\title{
New insights on the complex planetary nebula Hen 2-113 ${ }^{\star}$
}

\author{
E. Lagadec ${ }^{1}$, O. Chesneau ${ }^{2}$, M. Matsuura ${ }^{3}$, O. De Marco ${ }^{4}$, J. A. de Freitas Pacheco ${ }^{1}$, \\ A. A. Zijlstra ${ }^{3}$, A. Acker ${ }^{5}$, G. C. Clayton ${ }^{6}$, and B. Lopez ${ }^{2}$ \\ 1 Observatoire de la Côte d'Azur-CNRS-UMR 6202, Dept. Cassiopée, BP 4229, 06304 Nice, France \\ e-mail: lagadec@obs-nice.fr \\ 2 Observatoire de la Côte d'Azur-CNRS-UMR 6203, Dept. Gemini, Avenue Copernic, 06130 Grasse, France \\ 3 Department of Physics and Astronomy, University of Manchester, Sackville Street, PO Box 88, Manchester M60 1QD, UK \\ 4 American Museum of Natural History, Dept. of Astrophysics, Central Park West at 79th Street, New York NY 10024, USA \\ 5 Observatoire de Strasbourg, 11 rue de l'Université, 67000 Strasbourg, France \\ 6 Department of Physics and Astronomy, Louisiana State University, Baton Rouge, LA 70803, USA
}

Received 8 July 2005 / Accepted 22 August 2005

\section{ABSTRACT}

We report on infrared observations of the planetary nebula Hen 2-113 obtained with VLT/NACO, VLTI/MIDI, VLT/ISAAC and TIMMI at the ESO 3.6 m. Hen 2-113 exhibits a clear ring-like structure superimposed to a more diffuse environment visible in the $L^{\prime}(3.8 \mu \mathrm{m}), M^{\prime}(4.78 \mu \mathrm{m})$ and $8.7 \mu \mathrm{m}$ bands. No clear core at $8.7 \mu \mathrm{m}$ and no fringes through the $N$ band could be detected for this object with MIDI. A qualitative interpretation of the object structure is proposed using a diabolo-like geometrical model. The PAH content of the nebula was also studied with ISAAC and TIMMI observations. This indicates that the PAHs are mostly concentrated towards the lobes of the diabolo and the bipolar lobes of the nebula. In $L^{\prime}$ band, a void $0.3^{\prime \prime}$ in diameter was discovered with NACO around the central source. The $L^{\prime}$ and $M^{\prime}$ fluxes from the central source were derived from NACO data indicating an important infrared excess with respect to the expected stellar emission based on stellar models and short wavelength data. The observed flux from this source in the $L^{\prime}$ and $M^{\prime}$ is about 300 and 800 times respectively than those expected from a model including only the central star. Moreover, the central object appears resolved in $L^{\prime}$ band with measured $F W H M$ of 155 mas. This infrared excess can be explained by emission from a cocoon of hot dust $(T \sim 1000 \mathrm{~K})$ with a total mass $\sim 10^{-9} M_{\odot}$.

Key words. techniques: interferometric - techniques: high angular resolution - stars: AGB and post-AGB - stars: circumstellar matter stars: imaging - infrared: stars

\section{Introduction}

One of the most debated questions regarding the postAGB evolution of low and intermediate mass stars is the departure from spherical symmetry observed in circumstellar envelopes of Pre-Planetary Nebulae (PPNe) and Planetary nebulae (PNe). Whereas AGB stars have roughly spherical envelopes, many PNe exhibit axisymmetric structures or even more complex morphologies.

Many theoretical models have been proposed to explain the shapes of PPNe and PNe (see a recent review by Balick \& Frank 2002). Most theories dealing with the shaping of PNe and PPNe rely on the velocity field of circumstellar material distributed either in an expanding toroidal structure or in an accretion disk. The formation of such structures requires the presence of a binary companion, rotation and/or magnetic fields. During the past few years, various observations have

* Based on observations made with the Very Large Telescope Interferometer at Paranal Observatory under programs 073.D-0130, 074.D-0139. been conducted aiming to detect features associated with mechanisms responsible for the shaping of PNe. De Marco et al. (2004), as well as Sorensen \& Pollacco (2004), showed that a significant binary population may be present among the central stars of PNe. Detection of magnetic fields around AGB stars and central stars PNe has also been mentioned recently by different teams (Miranda et al. 2001; Etoka \& Diamond 2004; Bains et al. 2004; Jordan et al. 2005), but the field strengths are not enough to dominate the flow dynamics. Since the most extreme bipolar morphologies appear to be related to circumstellar disks (Balick 1987), the detection and study of disks close to central stars is a very active field of research. Presently, this field benefits particularly from high spatial resolution techniques like adaptive optics on $8 \mathrm{~m}$ class telescopes (see, for instance, Biller et al. 2005) and recently, from the advent of interferometry in the mid-infrared (MIR) wavelength range (Leinert et al. 2004). These new facilities give a complementary view of circumstellar disks discovered by the HST (Sahai \& Nymann 2000; De Marco et al. 2002; Meakin et al. 2003). A good example of such an approach is the study of the disk in 
NGC 6302 by Matsuura et al. (2005), using HST and VLT observations.

In this sense, the compact and young PNe Hen 2-113 (Hen 3-1044, PK 321+03.1, IRAS 14562-5406) is a good example of an object with a complex morphology which, in spite of its kinematical properties, is typical of a type IIb planetary nebula (Costa \& de Freitas Pacheco 1996), this type of PNe being mostly circular in shape (Phillips 2005). The central star (CS) has a Wolf-Rayet type spectra and was classified as a [WC10] by Crowther et al. (1998). CS exhibiting WR spectra represent about $10-15 \%$ of all CS (Górny \& Tylenda 2000).

The ISO spectra of Hen 2-113 show simultaneously the presence of C-rich and O-rich dust grains (Waters et al. 1998). The dual dust chemistry phenomenon in PNe appears to be correlated with the presence of cool [WC] type CS (Zijlstra et al. 1991; De Marco \& Soker 2002).

De Freitas Pacheco et al. (1993) carried out a spectral analysis of the wind of Hen 2-113, which led him to conclude that the carbon-to-helium ratio is $\mathrm{C} / \mathrm{He}=0.5$. This value is similar to that of 0.55 found in an independent way by De Marco \& Crowther (1998), who also derived a mass-loss rate of $3.8 \times 10^{-6} M_{\odot} \mathrm{yr}^{-1}$ for an estimated terminal velocity of $1000 \mathrm{~km} \mathrm{~s}^{-1}$. If the terminal velocity is revised downward $\left(V_{\infty} \sim 250 \mathrm{~km} \mathrm{~s}^{-1}\right)$, the resulting mass-loss rate would be decreased by a factor of 8 , since $\dot{M} \propto V_{\infty}^{3 / 2}$. This revised value is consistent with Leuenhagen, Hamann \& Jeffery's estimate (1996) of $3.4 \times 10^{-7} M_{\odot} \mathrm{yr}^{-1}$ while being smaller than the estimation of $3.9 \times 10^{-6} M_{\odot} \mathrm{yr}^{-1}$ reported by De Marco \& Crowther (1998).

From high resolution Coudé spectra, de Freitas Pacheco et al. (1993) decomposed the main interstellar components of the NaI doublet and, using a rotation model for the Galaxy, they derived a distance of $3.1 \mathrm{kpc}$ for Hen 2-113. A similar value ( $3.5 \mathrm{kpc}$ ) was obtained by Leuenhagen et al. (1996), but a lower distance $(1.2 \mathrm{kpc})$ was derived by De Marco \& Crowther (1998). The effective temperature of the CS was estimated to be 29000 K by De Marco \& Crowther (1998), who modeled the star's atmosphere. From the H-Zanstra and the Stoy methods, de Freitas Pacheco et al. (1993) derived a lower effective temperature, namely $T_{\text {eff }}=22300 \mathrm{~K}$, consistent with the absence of HeII lines in the wind and the low excitation nebular spectrum (de Freitas Pacheco et al. 1992; De Marco et al. 1997).

Despite the careful study of the fundamental parameters of the CSs and nebulae of Hen 2-113 and CPD-568032 by De Marco et al. (1997, 1998) and De Marco \& Crowther (1998), the morphology of the nebula of Hen 2-113 was poorly known until the HST observations by Sahai et al. (2000, hereafter SNW00). These authors showed that Hen 2-113 exhibits a complex geometry, roughly bipolar with two bright, knotty, compact ringlike structures around the central star. This compact structure is embedded in a larger and fainter spherically symmetric structure and SNW00 were puzzled to notice that the central star of Hen 2-113 was conspicuously offset from the geometrical centers of the rings and from the circular contours attributed to the formal AGB wind.

In the present work we report new observations on Hen 2-113 performed with different infrared imaging
Table 1. Journal of observations with NACO/UT4.

\begin{tabular}{|c|c|c|c|c|c|}
\hline Star & Filter & Camera & Time & $t_{\exp }$ & Seeing \\
\hline \multicolumn{6}{|c|}{ 04/05-05-2004, airmass $1.15-1.3$} \\
\hline Hen $2-113^{i}$ & $M^{\prime}$ & L27 & $02: 34: 48$ & $37 \mathrm{~s}$ & 0.74 \\
\hline HD $130572^{i}$ & $M^{\prime}$ & L27 & $03: 19: 34$ & $18 \mathrm{~s}$ & 1.1 \\
\hline \multicolumn{6}{|c|}{ 13/14-05-2004, airmass $1.15-1.20$} \\
\hline Hen $2-113^{i}$ & $L^{\prime}$ & L27 & 02:08:30 & $100 \mathrm{~s}$ & 0.42 \\
\hline \multicolumn{6}{|c|}{ 29/30-06-2004, airmass 1.15-1.20 } \\
\hline Hen $2-113^{i}$ & $L^{\prime}$ & L27 & $05: 17: 54$ & $40 \mathrm{~s}$ & 0.90 \\
\hline \multicolumn{6}{|c|}{ 04/05-07-2004, airmass 1.15-1.20 } \\
\hline Hen $2-113^{i}$ & $L^{\prime}$ & $\mathrm{L} 27$ & $23: 46: 22$ & $40 \mathrm{~s}$ & 0.93 \\
\hline Hen 2-113 & $L^{\prime}$ & L27 & $23: 59: 41$ & $40 \mathrm{~s}$ & 0.76 \\
\hline HD $130572^{i}$ & $L^{\prime}$ & L27 & $00: 21: 31$ & $200 \mathrm{~s}$ & 0.66 \\
\hline
\end{tabular}

${ }^{i}$ With neutral density filter.

instruments (ISAAC and TIMMI) and high spatial resolution techniques, namely adaptive optics with NACO/VLT. The infrared domain offers a unique opportunity to observe with great detail the bulk of the dust emission in the very central region of the object. In particular, we attempted to detect and study small scale structures in the MIR with the long baseline interferometer MIDI/VLTI. The object was over-resolved with $46 \mathrm{~m}$ baselines and no interferometric data could be recorded, but the acquisition images are used in our study. The remainder of this paper is organized as follows: in Sect. 2 the infrared observations are presented, in Sect. 3 we perform a comparative analysis between the infrared and optical HST images, in Sect. 4 a possible geometry for Hen 2-113 is discussed and, finally, in Sect. 5 the conclusions are given.

\section{Observations}

\subsection{NACO high resolution imaging}

We have observed Hen 2-113 with the adaptive optics camera NACO attached to the fourth $8.2 \mathrm{~m}$ Unit Telescope (UT) of the Very Large Telescope (VLT), European Southern Observatory (ESO) Paranal, Chile. NAOS was operated in the visual wavefront sensor configuration with the SBRC Aladdin $1024 \times 1024$ detector. We observed the target with $L^{\prime}(3.8 \mu \mathrm{m})$ and $M^{\prime}(4.78 \mu \mathrm{m})$ broad-band filters. Using camera mode L27, the field of view was $28^{\prime \prime} \times 28^{\prime \prime}$ and the pixel scale was 27.1 mas per pixel. The auto-jitter mode was used, that is, at each exposure, the telescope moves according to a random pattern in a $10^{\prime \prime}$ box. A cross-correlation technique was used to recenter the images at about 0.25 pixel accuracy.

Individual dithered exposures were co-added, resulting in a total exposure time $t_{\exp }$ shown in Table 1. The data reduction was performed using an IDL routine developed by us that processes the $L^{\prime}$ and $M^{\prime}$ individual frames as follows. First of all, for $L^{\prime}$ images, bad pixels are removed. The sky is then computed as the mean of the dithered exposures, and subtracted frame by frame. Finally, all the sky-subtracted frames are shifted and added together. The reduced broad-band images are shown in Fig. 1. For the $M^{\prime}$ chopped images, we subtracted 

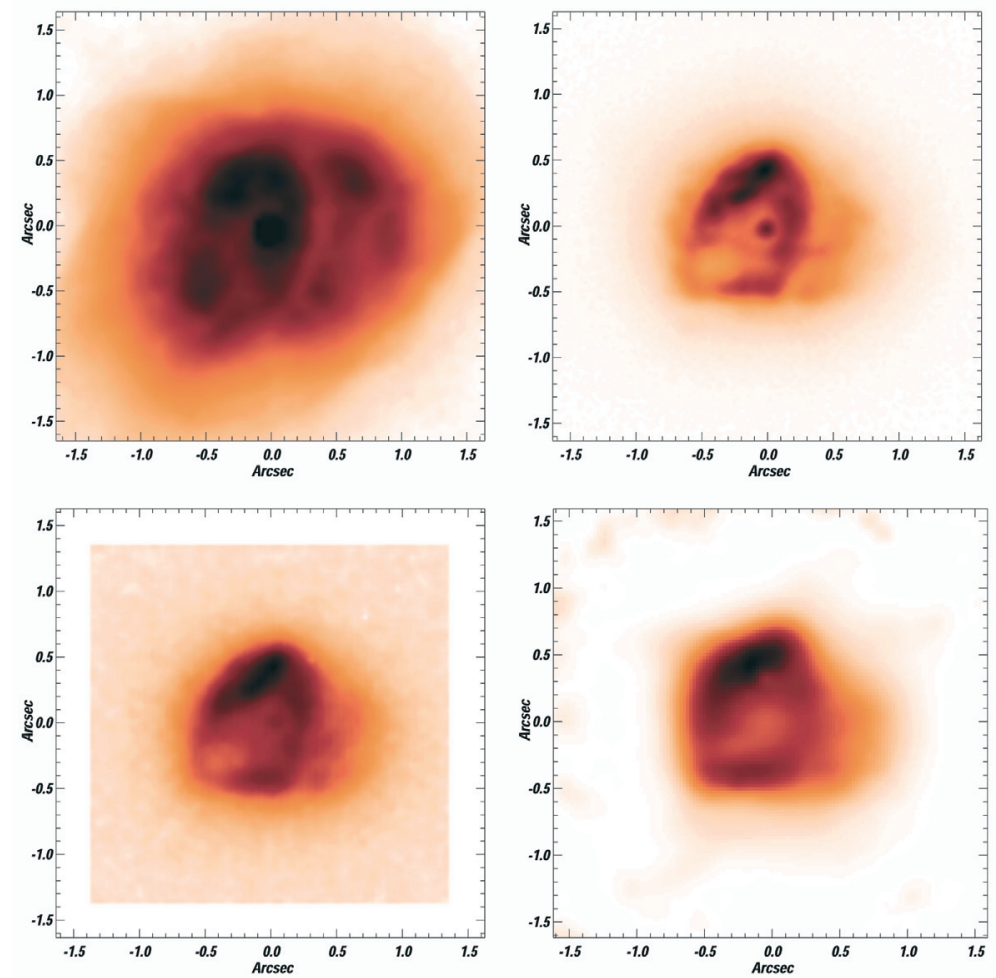

Fig. 1. Comparison of the HST image in H $\alpha$ (upper left), the NACO $L^{\prime}$ (upper right) and $M^{\prime}$ (lower left), and the deconvolved MIDI acquisition image at $8.7 \mu \mathrm{m}$. North is up and east to the left.

the sky. The spatial resolution of our $L^{\prime}$ image has been improved by a deconvolution with the Lucy-Richardson algorithm to reach $\sim 60$ mas (Fig. 2).

The star HD 130572 (A0V) was used to derive the point spread function (PSF) and as photometric standard. Its $J, H$ and $K$ magnitudes, from the 2MASS catalogue, are, respectively, $6.39 \pm 0.02,6.41 \pm 0.02,6.37 \pm 0.02$ and its $F W H M$ is $114 \pm$ 4 mas and $147 \pm 6$ mas in the $L^{\prime}$ and $M^{\prime}$ bands, respectively. The magnitude in the $L^{\prime}$ and $M^{\prime}$ bands was assumed to be 6.39 .

We estimated the magnitude of the full nebula to be $m_{L^{\prime}}=$ $4.1 \pm 0.2$ and $m_{M^{\prime}}=3.2 \pm 0.3^{1}$ in the $L^{\prime}$ and $M^{\prime}$ bands respectively. Then, we performed photometry of the central source using a method described in Chesneau et al. (2005), based on PSF subtraction techniques. The $L^{\prime}$ and $M^{\prime}$ radial means of the PSF and the source were computed, and the $L^{\prime}$ curves are shown in Fig. 3. The radial mean of the PSF was then scaled to the central object to evaluate its photometry, independently of the neighboring nebula, based on the assumption that this object is indeed unresolved. The $L^{\prime}$ and $M^{\prime}$ magnitudes of the central source of Hen 2-113 were estimated as $m_{L^{\prime}}^{\prime}=8.4 \pm 0.1 \mathrm{mag}$ and $m_{M^{\prime}}^{\prime}=8.3 \pm 0.2 \mathrm{mag}$, respectively. Interestingly, it appeared that the central source could not be considered as unresolved and could be fitted with an Airy curve having a FWHM of $155 \pm 16$ mas, i.e. close to the $M^{\prime}$ band spatial resolution. We stress that this result is based on the comparison of the radial curves from the four data sets recorded in $L^{\prime}$ band. The curves

${ }^{1}$ The quality of the $M^{\prime}$ images is quite limited in terms of noise and dynamic range leading to the large error bar for the integrated flux of the full nebula.

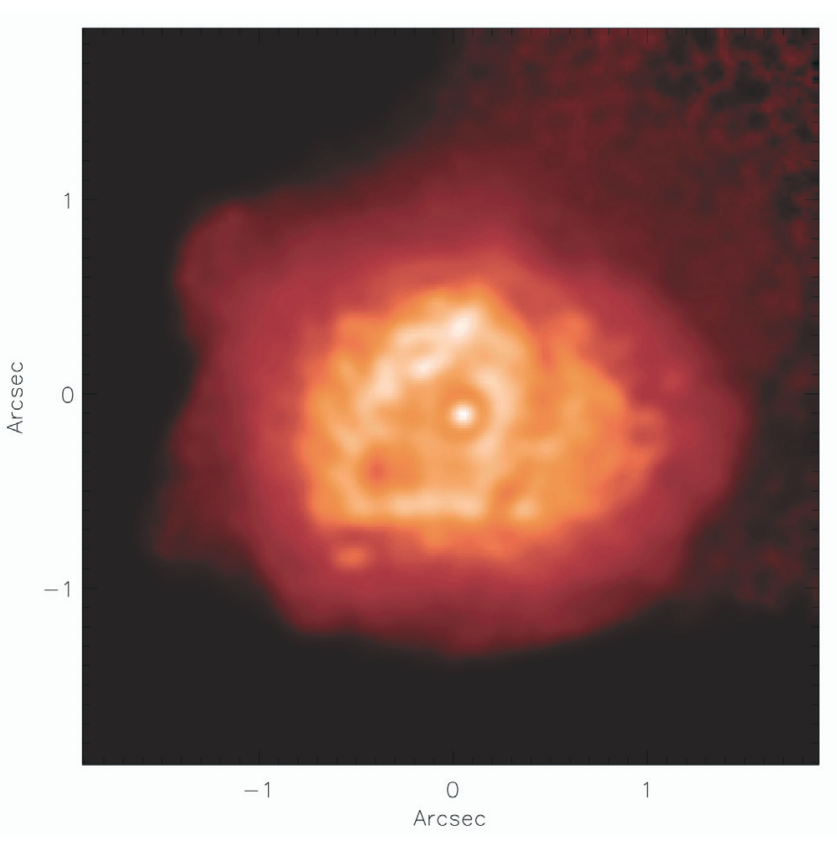

Fig. 2. NACO/VLT $L^{\prime}$ deconvolved image of Hen 2-113, log scaled in order to enhance the contrast. Dynamic range is $\sim 10^{4}$ and resolution $\sim 60$ mas. In the North/west, low level artefacts of deconvolution are visible. North is up and east to the left.

agree within about $4 \%$ and $2 \%$ at 0.27 and 0.54 arcsec respectively and span a seeing range from 0.42 to 0.93 arcsec. In the $M^{\prime}$ band, the central object is also extended and embedded in a diffuse environment (Fig. 3). 

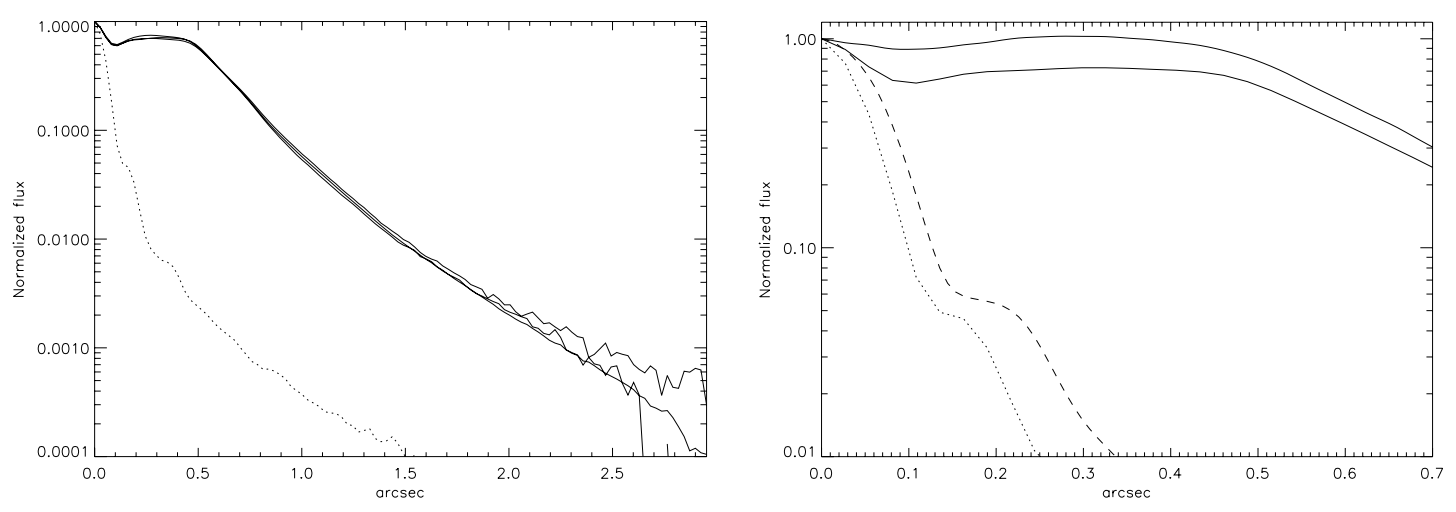

Fig. 3. Left: radial flux normalized to the peak (filter $L^{\prime}$ ) for the three different observations of Hen 2-113 (solid line), and the PSF HD 130572 (dotted line). Right: closer view of the $L^{\prime}$ (dotted) and $M^{\prime}$ (dashed) PSF curves compared to Hen 2-113 $L^{\prime}$ (lower) and $M^{\prime}$ (upper).

\subsection{MIDI acquisition image}

The VLTI/MIDI interferometer (Leinert et al. 2003, 2004) operates like a classical Michelson interferometer to combine the MIR light ( $N$ band, 7.5-13.5 $\mu \mathrm{m}$ ) from two VLT UTs. Observations of Hen 2-113 and CPD -568032 have been performed in 2004, April 08. The observations of CPD -568032 will be presented in a forthcoming paper (Chesneau et al. 2006, in preparation). The log of the observations is presented in Table 2. For these observations, the UT2 and the UT3 telescopes were used with a separation of $46.6 \mathrm{~m}$ and with the baseline oriented $40^{\circ}(\mathrm{E}$ of $\mathrm{N})$. This baseline provides a resolving power of the order of 40 mas and was adopted in order to detect compact dusty structures around the central star.

The observing sequence, typical of interferometric measurements is described in Leinert et al. (2003, 2004) and Przygodda et al. (2003). It must be stressed that the MIDI instrument detected no fringes as the target appeared resolved for a single dish $8 \mathrm{~m}$ telescope. Nevertheless, the acquisition images from this instrument were scientifically interesting and their reduction is described in the next section.

The chopping mode $\left(f=2 \mathrm{~Hz}\right.$, angle $\left.=-90^{\circ}\right)$ was used to visualize and to accurately point at the star, which is usually not perfectly centered in a first acquisition image, and is centered in a second step. Such a pointing accuracy is needed to get good quality interference pattern. The number of frames recorded for each image was generally 2000, and the exposure time per frame is by default $4 \mathrm{~ms}$ to avoid fast background saturation. If the pointing is not satisfactory, the procedure is started again. The default filter is centered at $8.7 \mu \mathrm{m}(1.6 \mu \mathrm{m}$ wide $)$. This filter has been used for the three acquisitions of the science targets and the calibrators. For overhead considerations, no nodding sequences were recorded.

It immediately appeared that Hen 2-113 was fully resolved with the 250 mas resolving power of a single-dish $8 \mathrm{~m}$ telescope. A few attempts to detect fringes on some emerging structures were conducted without success.

Custom software written in the IDL language was developed in order to reduce MIDI observations, including images and spectra extraction.

The first step of the reduction is to read in the acquisition datasets, average the frames on the target and the frames on
Table 2. Journal of observations with MIDI/UT2-UT3: acquisition images.

\begin{tabular}{llcrr}
\hline \hline Star & Name & Time & Frames & $t_{\text {exp }}$ \\
\hline HD 152786 & PSF01 & $04: 12: 39$ & 10000 & $100 \mathrm{~s}$ \\
HD 152786 & PSF02 & $04: 13: 41$ & 5000 & $50 \mathrm{~s}$ \\
HD 152786 & PSF03 & $04: 14: 36$ & 15000 & $150 \mathrm{~s}$ \\
HD 152786 & PSF04 & $04: 16: 28$ & 15000 & $150 \mathrm{~s}$ \\
HD 152786 & PSF05 & $04: 17: 50$ & 15000 & $150 \mathrm{~s}$ \\
Hen2-113 & HEN01 & $04: 50: 46$ & 2000 & $20 \mathrm{~s}$ \\
Hen2-113 & HEN02 & $04: 56: 44$ & 2000 & $20 \mathrm{~s}$ \\
Hen2-113 & HEN03 & $04: 59: 50$ & 2000 & $20 \mathrm{~s}$ \\
Hen2-113 & HEN04 & $05: 02: 12$ & 2000 & $20 \mathrm{~s}$ \\
HD 152786 & PSF06 & $05: 55: 34$ & 5000 & $50 \mathrm{~s}$ \\
HD 152786 & PSF07 & $05: 56: 22$ & 15000 & $150 \mathrm{~s}$ \\
HD 152786 & PSF08 & $05: 57: 11$ & 2000 & $20 \mathrm{~s}$ \\
HD 152786 & PSF09 & $05: 57: 58$ & 2000 & $20 \mathrm{~s}$ \\
HD 152786 & PSF10 & $05: 58: 53$ & 2000 & $20 \mathrm{~s}$ \\
HD 152786 & PSF11 & $09: 28: 54$ & 5000 & $50 \mathrm{~s}$ \\
HD 152786 & PSF12 & $09: 29: 45$ & 15000 & $150 \mathrm{~s}$ \\
\hline
\end{tabular}

the sky, and subtract the averaged sky-frame from the averaged target-frame. Despite the high number of optical elements in the VLTI/MIDI system ( 33 in total), the quality of the $8.7 \mu \mathrm{m}$ images is comparable to the best MIR images published to date, i.e. Eta Carinae (Chesneau et al. 2005). The angle subtended by a pixel on the sky is approximatively 98 mas and the North direction rotates during the observation.

Since MIDI is a long baseline interferometer, most of the targets are usually unresolved by a single $8 \mathrm{~m}$ telescope providing a wealth of instrumental PSF acquisitions.

The airmass of the targets varies between 1.2 and 1.8, the optical seeing was oscillating between 0.65 and 0.85 .

The spatial resolution has been slightly increased by performing a deconvolution using 30 iterations of the Lucy-Richardson algorithm and the result is shown in Fig. 1. The spatial resolution reached after the treatment is about 150 mas. The levels where the different deconvolved images begin to disagree are between $1 \%$ of the maximum flux of the 
Table 3. Filters and image quality and fluxes. The central wavelengths $\left(\lambda_{0}\right)$ and the widths of the filters are listed for ISAAC. $T_{\exp }$ is the exposure time. Infrared seeings are measured $F W H M$ of the nearby star (2MASS 14595478-5417453, USNO 0300-22661298).

\begin{tabular}{lccrc}
\hline \hline Band & $\begin{array}{c}\lambda_{0} \\
{[\mu \mathrm{m}]}\end{array}$ & $\begin{array}{c}\Delta \lambda \\
{[\mu \mathrm{m}]}\end{array}$ & $\begin{array}{r}T_{\exp } \\
{[\mathrm{min}]}\end{array}$ & $\begin{array}{c}\text { Infrared seeing } \\
{[\operatorname{arcsec}]}\end{array}$ \\
\hline NB_3.21 & 3.21 & 0.05 & 12 & 0.40 \\
NB_3.28 & 3.28 & 0.05 & 20 & 0.40 \\
\hline
\end{tabular}

image, depending on the quality of the recorded images. Due to different optical properties of the beam between UT2 and UT3, only the best UT3 images were used.

\subsection{ISAAC imaging}

Near-infrared images of Hen 2-113 were taken with the Infrared Spectrometer And Array Camera (ISAAC) at the VLT. The data were acquired over two nights in 2004, August 05 and 06 with UT1. The observations are summarized in Table 3. Calibration data were obtained with short exposures of Hen 2-113 and the photometric standard star HIP 76074. The images shown in this paper were obtained on the 5 th of August, and total fluxes were scaled to be consistent with those of the 6th of August data. The background was subtracted by chopping and nodding to a position $20^{\prime \prime}$ to the north/west, while keeping the source on the array. Jittering was used to improve flat-fielding and to minimise the influence of the pixelto-pixel variation. The pixel scale is $0.071^{\prime \prime}$. The resolution in the infrared is generally better than in optical by about $25 \%$ (Matsuura et al. 2004). We used Eclipse 4.1.2 for the datareduction. A detector's non-linearity correction is adopted as suggested by ISAAC instrument team.

Three nearby stars were used to calibrate the astrometry by comparing to their 2MASS positions (2MASS 14595265-5417418, 14595364-5417517, 145954365418162). The positions are consistent within 0.6 arcsec. The zero-point of the figures is RA $=14.59 \mathrm{~m} 53 \mathrm{~s} .4$, Dec $=$ $-54^{\circ} .18^{\prime} 7^{\mathrm{s}} .0$ (J2000).

For comparison with an optical image, we used $\mathrm{H} \alpha$ data obtained by Sahai et al. (2000) (Fig. 4). Distortion was corrected by the procedure of Anderson \& King (2003), and astrometry was performed using the coordinates of two nearby stars (14595364-5417517, 14595436-5418162). Even after the shift of HST and ISAAC images, there is an error of two ISAAC pixels found in the north/south direction. For image presentation, we used pipeline reduced data, and shifted the image according to the location of the central star. Image distortion is negligible within the central region of Hen 2-113.

\subsection{TIMMI imaging}

Observations were performed in 1995, February 05 and 07 at the ESO 3.6-m telescope in La Silla (Chile), using the midinfrared TIMMI camera (Lagage et al. 1993) with a detector consisting of a $64 \times 64$ pixel Ga:Si array. The resulting spatial

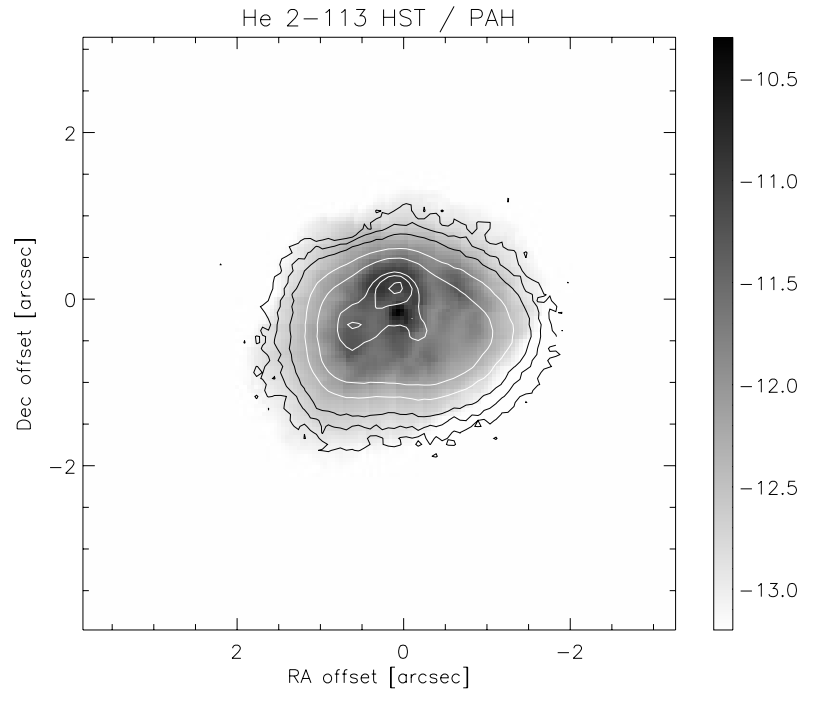

Fig. 4. HST F656 image and ISAAC $3.3 \mu \mathrm{m}$ PAH contour after continuum subtraction (NB_3.28 - NB_3.21). North is up and east to the left.

Table 4. Journal of observations with TIMMI/3.6 m.

\begin{tabular}{lllll}
\hline \hline Object & Filter & $\begin{array}{l}\lambda_{0} \\
(\mu \mathrm{m})\end{array}$ & $\begin{array}{l}\text { Filter width } \\
(\mu \mathrm{m})\end{array}$ & $T_{\exp }(\mathrm{s})$ \\
\hline Hen 2-113 & $\mathrm{N} 2$ & 9.78 & $9.14-10.43$ & 124.32 \\
Hen 2-113 & $\mathrm{N} 3$ & 12.55 & $11.80-13.30$ & 62.16 \\
Hen 2-113 & $\mathrm{SiC}$ & 11.65 & $10.30-13.00$ & 62.16 \\
HR 4763 & $\mathrm{N} 2$ & 9.78 & $9.14-10.43$ & 101.92 \\
HR 4174 & $\mathrm{N} 3$ & 12.55 & $11.80-13.30$ & 62.16 \\
HR 4174 & $\mathrm{SiC}$ & 11.65 & $10.30-13.00$ & 62.16 \\
\hline
\end{tabular}

scale of the system is 0.336 " $/$ pix. The log of the observations is given in Table 4 . These observations were made in a standard MIR observing mode, by chopping the secondary mirror and nodding the primary to subtract the background emission from the sky and telescope. The chopper throw was $18.3^{\prime \prime}$ toward the south and the nod beam position used was $18.3^{\prime \prime}$ north of the first position. To avoid saturation of the detector by the ambient photon background and to have good image quality, each individual nod cycle was split into many short exposures of $\sim 10 \mathrm{~ms}$. This procedure was repeated for as many cycles as needed to obtain the required total integration time. Nearly diffraction-limited images ( $\sim 0.7^{\prime \prime} F W H M$ for point sources) resulted from these short exposures. Filter wavelengths were selected in order to obtain information on the dust continuum as well as on spectral features like PAHs, while maximizing the detection sensitivity. The observations were carried out in four narrow-band filters, centered respectively at $9.78(\mathrm{~N} 2)$, $11.65(\mathrm{SiC})$ and $12.55 \mu \mathrm{m}(\mathrm{N} 3)$ and whose characteristics are given in Table 4.

The data reduction was performed using IDL selfdeveloped routines (Lagadec et al. 2005). Individually-chopped frames were spatially oversampled by a factor of 4 and shifted to the nearest 0.25 pixel by using a cross-correlation algorithm to correct for turbulent motions and flexure drifts. Images were 
then co-added to produce a single flat-field-corrected image for each filter, comprising the average of the chop and nod differences. Reference stars were observed and analyzed in the same way and were used to derive the instrumental PSF at each filter. Images were deconvolved by using the Richardson-Lucy algorithm.

\subsection{HST imaging}

In order to compare our infrared images with optical ones, a total of six $\mathrm{H} \alpha$ exposures of Hen 2-113 (F656N $(\lambda=$ $656.2 \mathrm{~nm}, \lambda=2.2 \mathrm{~nm}$ ), taken as part of an HST SNAPshot imaging program (GO program 8345, Sahai et al.), were retrieved from HST archives and processed via the standard calibration pipeline. The nebula lies entirely within the field of the Planetary Camera $(800 \times 800$ pixels; pixscale $=0.0456^{\prime \prime}$ pixel $^{-1}$ ) of WFPC2. The image was then rotated for comparison with the other instruments.

These images (Fig. 1) have been extensively described in SNW00 and will be used in the following section for comparison with our observations.

\section{Optical versus infrared morphology}

The optical nebula exhibits a bipolar shape $\left(\mathrm{PA}=136^{\circ}\right)$. The diffuse lobes have a radial extent of about $2^{\prime \prime}$ and are well defined with sharp edges. Superimposed on this diffuse environment, a brighter region is composed of two elliptical rings. The second ring (ring 2) is fainter and incomplete, but its structure is well-defined and it appears coaxial to the first one (ring 1). The central star of Hen 2-113 is conspicuously offset from the geometrical centers of the rings and is also not located symmetrically between these centers. It is also offset from the midpoint of the line joining the tips of the main NW-SE lobes of the nebula. (See Figs. 1a,c and 3 in SNW00)

The NACO and MIDI (Figs. 1, 2, 5, 6) high resolution images show a nebula whose extension is limited to the bright core of HST images. The limits of this core are as reported by De Marco et al. (1997) about $1.4^{\prime \prime} \times 1.1^{\prime \prime}$.

The star, visible in the $L^{\prime}$ and $M^{\prime}$ NACO images disappears in the $N$ band (MIDI $8.7 \mu \mathrm{m}$ ). A bright ring-like knotty structure which coincides with ring 1 observed by SNW00 is also observed in the $L^{\prime}, M^{\prime}$ NACO images, and in the MIDI $8.7 \mu \mathrm{m}$ deconvolved image. This ring is roughly elliptical and the central star is offset from the geometrical center of the ring of $0.15^{\prime \prime}$ along PA $\sim 65^{\circ}$. Its major axis has an extension of $\sim 1.2^{\prime \prime}$ and a minor-to-major axis ratio of $\sim 0.6$ and the line passing through the center of the ring and perpendicular to its major axis is orientated at a PA of $\sim 65^{\circ}$. Ring 2 is less clearly visible and is replaced by an elongated region of diffuse emission from $L^{\prime}$ images to $N$ band images. These rings are thus smaller in our mid-infrared observation (Fig. 1) than the one observed with HST. The three color composite image (Fig. 6) indeed shows that the rings observed at mid-infrared wavelenghts lie inside those observed in $\mathrm{H}_{\alpha}$.

The dark lane (0.2" width in $L^{\prime}$ and HST images) in the $L^{\prime}$ images which splits the emission between the western border of the bright ring and this elongated diffuse emission is

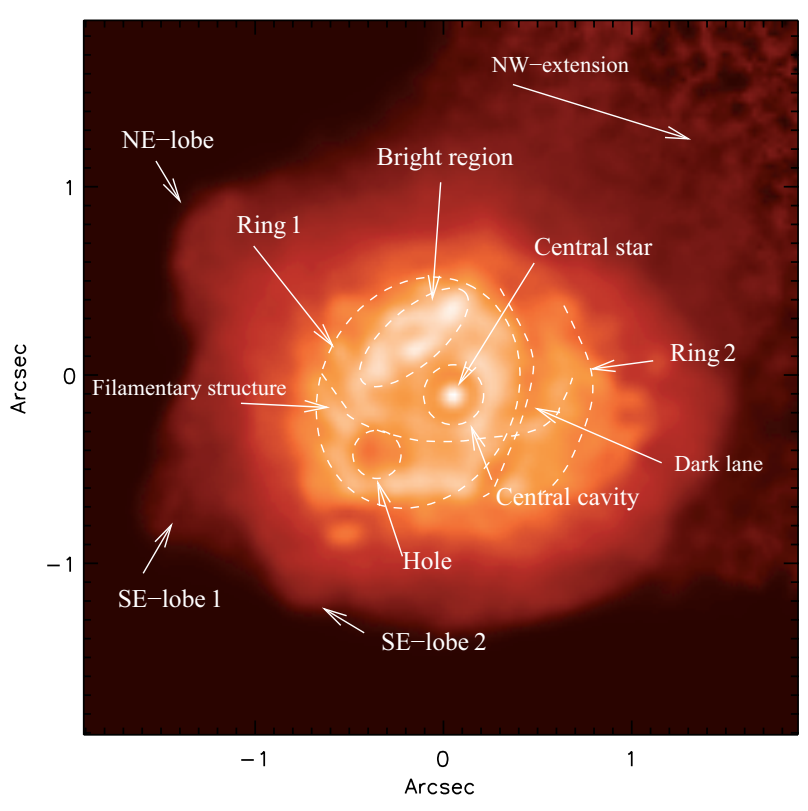

Fig. 5. Schematic view of Hen 2-113 overlaid on the Naco $L^{\prime}$ image. North is up and east to the left.

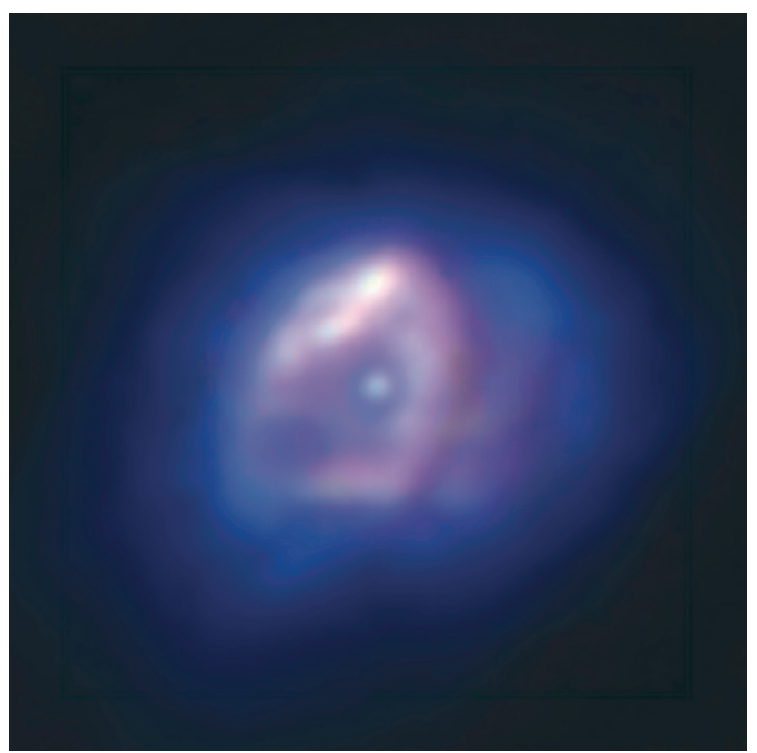

Fig. 6. Three color composite image of Hen2-113 made by combining HST $\mathrm{H}_{\alpha}$ (blue), NACO $L^{\prime}$ (green) and $M^{\prime}$ (red) images. North is up and east to the left.

coincident with the HST one (Fig. 6). The lane can be distinguished in the $M^{\prime}$ and $8.7 \mu \mathrm{m}$ images but the reduction of the spatial resolution at these wavelengths together with the decrease of optical thickness prevent from a clear identification of this structure. Such a dark lane suggests the presence of a ring of cold dust.

A bright region is observed on the North-East of ring 1 in our NACO $L^{\prime}$ and $M^{\prime}$ image as in MIDI $8.7 \mu \mathrm{m}$ image. This blob may represent emission from dust in excess in this part of ring 1 . It is 2 or 3 times brighter than the other parts of ring 1 in $\mathrm{H}_{\alpha}, L^{\prime}, M^{\prime}$ and MIDI images, and its dimension is roughly $0.7^{\prime \prime} \times 0.2^{\prime \prime}$. 
A small circular region of low emissivity (hole), probably due to a lack of material, is detected at $\sim 0.5^{\prime \prime}$ to the South-East of the central star along a PA $\sim 128^{\circ}$ in our two NACO images, and can be distinguish with the HST and MIDI observations. Its diameter is roughly $0.3^{\prime \prime}$. It is interesting to note that this direction nearly coincides with the orientation of the bipolar nebula surrounding the two rings as observed by SNW00 $\left(\mathrm{PA}=136^{\circ}\right)$. Furthermore, our deconvolved NACO $L^{\prime}$ image seems to show the presence of material on the North-West, alignated with the direction formed by the central star and the hole. Another possible interpretation would be the presence of a fairly dense clump of dust as observed in the PN Roberts 22 (Sahai et al. 1999). Nevertheless in this case we would not expect to see the bright blob in $\mathrm{H}_{\alpha}$ as seen in the HST image.

More complex structures are seen in the high dynamics $L^{\prime}$ NACO deconvolved image (Fig. 2). In particular ring 1, the lane and ring 2 seem to be broken by a thin emissive filamentary structure which can be traced to the eastern rim of ring 1 , passing $\sim 0.2^{\prime \prime}$ south from the star. This $0.1^{\prime \prime}$ wide filamentary structure is roughly perpendicular to the dark lane.

This image also shows that the bright core (the two rings and the lane) is surrounded by a faint low emission region of $2.3^{\prime \prime} \times 3^{\prime \prime}$. The brightest region of ring 1 is one hundred times brighter than this "halo". Three lobes are seen extending from this region. The brightest (NE-lobe) is orientated along a $\mathrm{PA} \sim 55^{\circ}$, coincident with the NE-lobe described by SNW00, supporting the reality of the fine structures observed in our deconvolved NACO image. Another lobe (SE-lobe 1) is observed in the SE direction, oriented along $\mathrm{PA} \sim 115^{\circ}$. The counterpart of this lobe could be the material seen on the north/west. The smallest lobe (SE-lobe 2) is also orientated roughly SE, with a $\mathrm{PA} \sim 142^{\circ}$.

A diminution of dust emission is detected close to the CS (Figs. 2, 3, 5), which is placed in a cavity of radius $\sim 0.3^{\prime \prime}$. Such a cavity is not seen in the $\mathrm{H} \alpha$ images obtained with the HST, suggesting that ionized gas fills the cavity or is seen in front of it.

\section{Interpretation}

\subsection{The structure of Hen2-113}

HST observations (SNW00), show that Hen 2-113 has an overall bipolar shape and two ringlike structures around the CS. SNW00 noticed also that the CS was offset from the geometrical center of the rings. This is confirmed by our NACO and MIDI observations and motivated us to make a simple geometrical model to explain it.

To disentangle projection effects and to understand the real three dimensional structure of an astronomical object is always a difficult task. First, we notice that ring 1 (eastern ring) is brighter than ring 2 (western ring), suggesting that ring 1 is closer than ring 2. A three color composite image (Fig. 6) seems to confirm this hypothesis. Indeed, the superposition of our $L^{\prime}$ and $M^{\prime}$ images with the $\mathrm{HST} \mathrm{H}_{\alpha}$ image indicates that the two rings are the projection of a diabolo-like torus (Fig. 7), with the eastern ring (top of the torus) pointing toward us. In such a geometry, the brightness difference of the two rings is

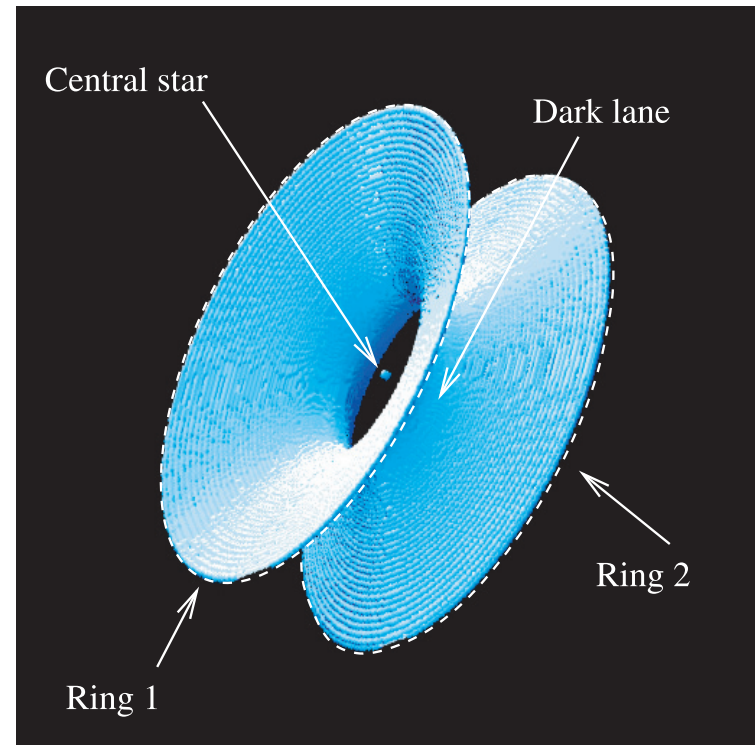

Fig. 7. Diabolo model with the adopted inclination parameters to visually fit the morpholgy observed with the HST.

easily explained by a screening effect from the equatorially enhanced dust density. The "diabolo-like" surface is represented by a revolution hyperboloid defined by the equations:

$$
\begin{aligned}
& x=a\left(1+u^{2}\right)^{n} \cos v \\
& y=a\left(1+u^{2}\right)^{n} \cos v \\
& z=c u
\end{aligned}
$$

with: $-\frac{\pi}{2} \leq u \leq \frac{\pi}{2}$ and $-\pi \leq v \leq \pi$.

The parameter $a$ fixes the radius of the diabolo, $c$ is the height and $n$ the opening angle. The code, written in IDL, produces an hyperboloid, which can be scaled according to its distance and inclination with respect to the plane of the sky, allowing a direct comparison with the observed images. The fit of the parameters was performed visually, searching for the best superposition between the simulated and the observed images, the HST optical image being the reference, as ring 2 is best seen on HST $\mathrm{H}_{\alpha}$. The aim of this model is to reproduce the main morphological features observed for the rings: the major-to-minor axis ratio of ring 1 is $\sim 2$, two thirds of ring 2 is observed, the central star is offset with respect to the center of the rings and the projection of the common axis of these rings on the skyplane is oriented with a PA of $\sim 65^{\circ}$.

Figure 7 shows a representation of our model. A satisfying superposition was obtained with a diabolo having an inclination $i=37^{\circ}$ and a PA $\sim 65^{\circ}, n=0.9$ and $a / c=3 / 2$. This geometrical model can explain the offset of the CS from the center of ring 1, but the offset from the center of the nebula (SNW00) is not explained by this geometry. It can be explained by proper motion of the star at $\sim 1 \mathrm{~km} \mathrm{~s}^{-1}$ with respect to the nebula.

The diabolo-shaped structure is tilted with respect to the bipolar nebula observed by SNW00, oriented along PA $=136^{\circ}$. Notice that the orientation of the diabolo $\left(\mathrm{PA} \sim 65^{\circ}\right.$ ) is coincident with none of the other features observed inside the nebula. 
SNW00 already noticed that the shaping of this nebula could not be explained with the generalized interacting stellar wind model (GISW) (Kwok et al. 1978; Balick 1987) as this model cannot explain multipolar morphologies. They proposed that the observed morphology could be explained if fast, collimated outflows change their direction with time (Sahai \& Trauger 98, hereafter ST98). Our observations strengthen the fact that the observed morphology cannot be explained by the GISW model as the morphology of the nebula is multipolar. The observed tilt also seems to be difficult to explain by the ST98 model. The hole observed in the south/east of the nebula could be a clue to our understanding of this structure. This hole is probably due to a lack of dust in this part of the nebula and it is interesting to notice that the direction formed by the CS and the hole $\left(\mathrm{PA} \sim 128^{\circ}\right.$ ) is nearly coincident with the orientation of the main bipolar nebula $\left(\mathrm{PA} \sim 136^{\circ}\right)$. This is seen clearly in our three color image (Fig. 6).

Since the nebula around Hen 2-113 is relatively young, it is only partially ionized, as the ionization front is still propagating inside the shell. The total measured $\mathrm{H} \beta$ flux (Acker et al. 1992) corrected by an extinction $E(B-V)=1.0$ (De Marco et al. 1997) is $F(\mathrm{H} \beta)=4.08 \times 10^{-11} \mathrm{erg} \mathrm{cm}^{-2} \mathrm{~s}^{-1}$. In this case, the ionized mass is given by

$M_{\text {ion }}=4 \pi D^{2} \frac{F(\mathrm{H} \beta) m_{\mathrm{p}}}{j(\mathrm{H} \beta)\left\langle n_{\mathrm{e}}\right\rangle}=\frac{31 D_{\mathrm{kpc}}^{2}}{\left\langle n_{\mathrm{e}}\right\rangle} M_{\odot}$

where $D$ is the distance, $j(\mathrm{H} \beta)$ is the line emissivity coefficient, $m_{\mathrm{p}}$ is the proton mass and $\left\langle n_{\mathrm{e}}\right\rangle=10^{5} \mathrm{~cm}^{-3}$ is the mean electron density in the nebula (de Freitas Pacheco et al. 1992). For distances in the range $1-3 \mathrm{kpc}$, the ionized mass is in the range $(0.3-2.8) \times 10^{-3} M_{\odot}$. For PNe, the mass of the ionized shell is anti-correlated with the mean electron density (Pottasch 1984). This can be understood by the fact that, as the ionization front propagates increasing the ionized mass, the shell expands and the density decreases. For a young nebula with a density of about $10^{5} \mathrm{~cm}^{-3}$, the expected ionized mass is about (2-4) $\times 10^{-3} M_{\odot}$ (Pottasch 1984), consistent with our findings and supporting the idea that Hen 2-113 is a young nebula. Note that SNW00 estimated that the expansion age of Hen 2-113 is $<550 \mathrm{yr}$.

\subsection{Infrared excess from circumstellar material close to the CS}

As mentioned in Sect. 2.1, the photometry of the CS gives $m_{L^{\prime}}^{\prime}=8.4 \pm 0.1 \mathrm{mag}$ and $m_{M^{\prime}}^{\prime}=8.3 \pm 0.2 \mathrm{mag}$ for filters $L^{\prime}$ and $M^{\prime}$, respectively. If we compare these values with predictions from atmosphere models, it appears that a star with the same effective temperature as Hen 2-113 should not be so bright at those wavelengths.

Indeed, using Kurucz models (Kurucz 1979) with $T_{\text {eff }}=$ $29000 \mathrm{~K}$ (De Marco \& Crowther 1998), we have estimated $L^{\prime} \sim 14.5$ and $M^{\prime} \sim 15.5$, by adopting $J=9.78$ (Webster \& Glass 1974, 2MASS $=9.51 \pm 0.026$ ) and assumining no extra light contribution to the CS emission at this wavelength. This assumptiandon seems to be justified by the analysis of continuum emission from the UV to the far-IR (see Fig. 1 by De Marco \& Crowther 1998). Therefore, the observed flux from the central object in the $L^{\prime}$ and $M^{\prime}$ bands is about $\sim 300$ and $\sim 800$ times higher respectively than expected from a model including only the central star. It is worth mentioning that a comparison between the PSF and the light profile of the bright central object indicates that the latter is resolved by our data, having an angular diameter of about 155 mas in the $L^{\prime}$ band, corresponding to dimensions of 140-430 $\mathrm{AU}$, if the distance of Hen 2-113 is in the range $1-3 \mathrm{kpc}$. Notice that a similar infrared excess was also observed by Matsuura et al. (2004) associated to the emission of the CS of NGC 6302.

We tested several hypothese to explain this small and intense emission, in particular free-free and dust emission.

In the hypothesis that the observed infrared excess results from bremsstrahlung, the required ionized gas mass within the central region is $M \sim 7 \times 10^{-5}-4 \times 10^{-4} M_{\odot}$ (adopting $T_{\mathrm{e}}=10000 \mathrm{~K}$ and $n_{\mathrm{e}}=10^{5} \mathrm{~cm}^{-3}$, de Freitas Pacheco et al. 1993) comparable to the present mass of ionized gas estimated for the whole nebula in Sect. 4.1. Thus this hypothesis seems implausible.

We then considered the possibility that the infrared excess is produced by a "cocoon" of hot amorphous carbon dust.

The wind of the CS of Hen 2-113 is C-rich and one could expect the formation of carbon dust grains as the envelope cools in the expansion and is decelerated by the material previously ejected. If re-radiation by dust is the origin of the infrared excess observed in the $L^{\prime}$ and $M^{\prime}$ bands, the mass of grains $M_{\mathrm{d}}$ inside a radius of 70-210 $\mathrm{AU}$, within the optically thin approximation, is

$M_{d}=\frac{4 \pi D^{2} f_{\lambda} a \delta}{3 Q_{\lambda} B_{\lambda}\left(T_{\mathrm{d}}\right)}$

where $f_{\lambda}$ is the observed flux in $L^{\prime}$ (or $M^{\prime}$ ) band, $a$ is the mean grain radius, $\delta$ is its density and $Q_{\lambda}$ is the absorption (emission) efficiency factor.

The grain temperature, $T_{\mathrm{d}}$, can be estimated from the flux ratio at two different wavelengths $(3.8 \mu \mathrm{m}$ and $4.78 \mu \mathrm{m}$ in the present case), e.g.,

$\frac{f_{\lambda_{1}}}{f_{\lambda_{2}}}=\frac{Q_{\lambda_{1}} B_{\lambda_{1}}\left(T_{\mathrm{d}}\right)}{Q_{\lambda_{2}} B_{\lambda_{2}}\left(T_{\mathrm{d}}\right)}$

Assuming dust grains of amorphous carbon $\left(\delta=2.0 \mathrm{~g} \mathrm{~cm}^{-3}\right)$ with $a=0.2 \mu \mathrm{m}$, a temperature of $\sim 1000 \mathrm{~K}$ is derived from the equation above. This high temperature would be expected for carbon grains close to the central star, not very far from the inner regions hotter than $1500 \mathrm{~K}$, when sublimation of amorphous carbon occurs. Once the temperature is known, the dust mass can be estimated from Eq. (2) and, for the range of distances, $D$, considered, one obtains $M_{\mathrm{d}} \sim$ (7-28) $\times 10^{-10} M_{\odot}$ and gas masses about two orders of magnitude higher ${ }^{2}$. The required dust masses imply optical depths of about $10^{-3}$ around $4 \mu \mathrm{m}$, justifying our optically thin approximation. These values, which represent a few years of mass-loss, are not in conflict with the nebular ionized gas mass estimated

2 This a lower limit for the mass. The physical conditions encountered so close to the CS would probably lead to a dust-to-gas mass ratio lower than $1 \%$. 
in Sect. 4.1 and the wind crossing time of the small circumstellar region considered is also a few years.

We note that the temperature and dust mass estimated here are remarkably consistent with the mass and temperature independantly estimated by SNW00 for their hot dust component by SED fitting and by De Marco \& Crowther (1998) by fit to the IR excess.

\subsection{PAHs}

Hen 2-113 belongs to a group of bipolar post-AGB stars at the center of young ionised PNe showing both PAH bands and crystalline silicates. A scenario proposed by Waters et al. (1998) and Molster et al. (1999) explains the observed dual chemistry observed around these stars. In this scenario, a disk, formed while the star was still oxygen-rich, remains during the high mass-loss phase when the star is carbon-rich, forcing the matter outflow to a bipolar geometry perpendicular to the direction of the disc.

Thus, we expect to observe different spatial distributions for PAHs and O-rich grains. Unfortunately, O-rich dust spectral features are observed at wavelengths $>15 \mu \mathrm{m}$ where atmospheric absorption makes ground-based observations very difficult. Observations at longer wavelengths (space-based, or with new MIR detectors) where the O-rich dust features lie would certainly bring new insights on the spatial distribution of $\mathrm{O}$ and C-rich dust.

Mid-infrared spectra of Hen 2-113 display strong features that can be attributed to PAHs. Different filters were used to observe a varying and significant amount of emission from these PAHs. The underlying continuum was estimated based on the work of Cohen et al. (2002), who represented the data by the superposition of a blackbody having a temperature of $395 \mathrm{~K}$ and that of a grey body $\left(\lambda^{-1.2} B_{\lambda}(T)\right)$. The results by Cohen et al. are comparable to those by SNW00, who fitted the continuum by three blackbody curves, cold $\left(T_{\mathrm{c}}=100 \mathrm{~K}\right)$, warm $\left(T_{\mathrm{w}}=378 \mathrm{~K}\right)$ and hot $\left(T_{\mathrm{h}}=900 \mathrm{~K}\right)$. We retrieved the ISO spectra of Hen2-113 from the archives and substracted it from the continuum modeled by two blackbodies. We then assume that the features resulting from this substraction was due to PAH. We then compare this with the filter transmissions to derive the $\mathrm{PAH}$ contribution in each filter.

The estimated $\mathrm{PAH}$ contributions in the different filters are $54 \%, 37 \%, 4 \%, 20 \%$ and $11 \%$ for ISAAC $3.28 \mu \mathrm{m}$, $\mathrm{N} 8.7 \mu \mathrm{m}, \mathrm{N} 2, \mathrm{SiC}$ and N3, respectively. These estimates are based on the assumption that the ISO fluxes recorded with large apertures are equivalent to the fluxes recorded with the various instruments used in this work. To study the morphology of Hen 2-113 in different filters quantitatively we fitted our images with a 2-dimensional Gaussian function. The bestfit parameters are summarized in Table 5. The observations in the PAH bands (ISAAC $3.28 \mu \mathrm{m}$, MIDI $8.7 \mu \mathrm{m}$ and TIMMI $\mathrm{SiC}$ filter) have a stronger major-to-minor axis ratio and tend to be oriented roughly east/west (except the MIDI image which has a $\mathrm{PA} \sim 68^{\circ}$ ).

To check if the PAHs and dust grains responsible for the continuum emission have different spatial distributions,
Table 5. Results of the fits to our image with a 2-dimensional Gaussian ellipse. $a / b$ is the ratio between the long and short axis of the ellipse, and $\theta$ is its orientation.

\begin{tabular}{lccc}
\hline \hline Filter & $a / b$ & PA $\left(^{\circ}\right)$ & PAH content $(\%)$ \\
\hline $8.7 \mu \mathrm{m}$ & 1.09 & 68 & 37 \\
$\mathrm{~N} 2$ & 1.05 & - & 4 \\
$\mathrm{SiC}$ & 1.16 & 91 & 20 \\
$\mathrm{~N} 3$ & 1.05 & - & 11 \\
$\mathrm{NB} \_3.21$ & 1.06 & 91 & - \\
$\mathrm{NB} \_3.28$ & 1.16 & 91 & 54 \\
Isaac subtract. & 1.34 & 92 & 100 \\
\hline
\end{tabular}

The orientation having no meaning for spherical objects, we did not mention angles for $\mathrm{N} 2$ and $\mathrm{N} 3$ filters.

we compared observations made with filters in the continuum and filters where the PAH contribution is expected to be significant. We thus compared ISAAC $3.21 \mu \mathrm{m}$ (continuum) and $3.28 \mu \mathrm{m}$ (PAH contribution $\sim 54 \%$ ) images as well as TIMMI N2 (continuum) and MIDI $8.7 \mu \mathrm{m}$ (PAH contribution $\sim 37 \%$ ) observations. Note that MIDI and TIMMI have different resolutions, so the MIDI observations were artificially degraded to the TIMMI resolution $(\sim 0.7 \mu \mathrm{m})$ by simple convolution with a 2D Gaussian to make these observations comparable.

Both ISAAC images show a single bright blob at the centre and a halo surrounding the center. Both blobs have an elongation toward the south/east and south/west direction. The halo is almost symmetric with a slight elongation, a bright blob at the center and a nearly spherical halo. The TIMMI N2 (continuum) and MIDI $8.7 \mu \mathrm{m}$ images also have a similar morphology. However, we can note that on the N2 image the object is broader than on the $8.7 \mu \mathrm{m}$ image. Whether this is due to the fact that the observations were made with different instruments and exposure times or wether the dust grains responsible for the continuum emission have a broader spatial distribution than PAH dust grains is hard to check.

Thus, we focused our work on the comparison of both ISAAC images by comparing radial cuts obtained with different filters (PAHs and continuum). First, using azimuthal averaging of these radial cuts, we note that in the PAH image the object is broader than on the continuum image. We then tried to check if there was some preferential direction for the broadening of the PAH image. We find that the strongest asymmetry (i.e., the intensity ratio for which a given radius is maximum) is observed for a $\mathrm{PA} \sim 139^{\circ}$, the intensity ratio being $\sim 1.55$. The strongest mean asymmetry (i.e. mean value of the intensity ratio) is observed for $\mathrm{PA} \sim 248^{\circ}$ (the intensity ratio is $\sim 1.24$ ).

Finally, our ISAAC observations show that PAHs seem to have a broader spatial distribution than the grains responsible for the continuum emission. The largest difference between the ISAAC PAH and continuum images are observed for $\mathrm{PA} \sim 139^{\circ}$ and $\sim 248^{\circ}$. It is interesting to notice that these orientations correspond to the orientations of the main bipolar nebula and the side of the diabolo-shaped structure pointing away from us. These structures being the youngest structures 
of the nebula, this tends to confirm the fact that the PAHs have been formed more recently than the dust grains responsible for the continuum emission.

\section{Conclusion}

In this paper we report high-spatial resolution infrared observations of the young PN Hen 2-113 obtained with different instruments at ESO, Chile (NACO, MIDI, ISAAC and TIMMI). These observations provide new insights on the complex morphology and structure of the nebula around it.

These observations, made possible by using the high resolution obtained with adaptive optics on VLT (NACO), led to the discovery of features previously unseen in this nebula and which seem difficult to explain with current models for the shaping of PNe. We discovered a void of $\sim 0.3^{\prime \prime}$ in diameter around the central source and a hole in the South-East of the nebula. The fact that the direction formed by the CS and this hole is nearly coincident with the orientation of the main axis of the nebula could be a clue for the understanding of the structure of the nebula. A simple geometrical model indicates that the CS is surrounded by a diabolo-shaped dusty structure which is tilted with respect to the rest of the nebula.

Our infrared data indicate an infrared excess with respect to the expected stellar emission based on stellar models and shorter wavelength data. We tested different hypotheses to explain this infrared excess. Dust emission very close to the CS seems to be responsible for this excess. Indeed, simple calculations indicate that emission from hot dust $(T \sim$ 900-1000 K) with mass $\sim 10^{-9} M_{\odot}$ can account for the infrared excess. The fact, that in their modeling of the SED of Hen 2-113, SNW00 need a hot dust component with the same mass and same temperature, strengthens the hypothesis that the infrared excess is due to emission from hot dust. We also note that such an infrared excess has been observed in at least one similar object (NGC 6302, Matsuura et al. 2005), indicating that this could be a feature common to this class of object. One would need high-angular resolution observations of other $\mathrm{PNe}$ to confirm this hypothesis.

These infrared observations, obtained with different instruments and wavelengths, allowed us to study the spatial distribution of PAHs in the nebula of Hen 2-113, known for displaying strong features attributed to PAHs. These observations indicate that the images made with filters containing PAH features seem to be broader than the one observed with continuum filters. The ISAAC observations indicate that the $\mathrm{PAH} /$ continuum difference is stronger along the direction of the main nebula and the diabolo-shaped torus. However, it is difficult with these observations to verify the scenario proposed by Waters et al. (1998) and Molster et al. (1999) for explaining the dual chemistry in this class of nebulae. Observations at longer wavelengths $(>15 \mu \mathrm{m})$, where spectral features of O-rich dust are observed, would thus be necessary to compare the spatial distribution of PAHs and O-rich dust.

Acknowledgements. The authors warmly thank those who made possible these service mode observations, in particular the ESO night astronomers for their careful work. ODM would like to acknowledge Janet Jeppson Asimov for financial support.

\section{References}

Acker, A., Marcout, J., Ochsenbein, F., et al. 1992, Garching: European Southern Observatory

Anderson, J., \& King, I. R. 2003, PASP, 115, 113

Bains, I., Richards, A. M. S., Gledhill, T. M., et al. 2004, MNRAS, 354,529

Balick, B. 1987, AJ, 94, 671

Balick, B., \& Frank, A. 2002, ARA\&A, 40, 439

Biller, B. A., Close, L. M., Li, A., et al. 2005, ApJ, 620, 450

Chesneau, O., Min, M., Herbst, T., et al. 2005, A\&A, 435, 1043

Chesneau, O., Collioud, A., \& de Marco, O. 2006, A\&A, in preparation

Cohen, M., Barlow, M. J., Liu, X.-W., et al. 2002, MNRAS, 332, 879

Costa, R. D. D., \& Pacheco, J. A. D. 1997, Planetary Nebulae, IAU Symp., 180, 217

Crowther, P. A., De Marco, O., \& Barlow, M. J. 1998, MNRAS, 296, 367

de Freitas Pacheco, J. A., Maciel, W. J., \& Costa, R. D. D. 1992, A\&A, 261, 579

de Freitas Pacheco, J. A., Costa, R. D. D., de Araujo, F. X., et al. 1993, MNRAS, 260, 401

De Marco, O., \& Crowther, P. A. 1998, MNRAS, 296, 419

De Marco, O., \& Soker, N. 2002, PASP, 114, 602

De Marco, O., Barlow, M. J., \& Storey, P. J. 1997, MNRAS, 292, 86

De Marco, O., Storey, P. J., \& Barlow, M. J. 1998, MNRAS, 297, 999

De Marco, O., Barlow, M. J., \& Cohen, M. 2002, ApJ, 574, L83

De Marco, O., Bond, H. E., Harmer, D., et al. 2004, ApJ, 602, L93

Etoka, S., \& Diamond, P. 2004, MNRAS, 348, 34

Górny, S. K., \& Tylenda, R. 2000, A\&A, 362, 1008

Jordan, S., Werner, K., \& O'Toole, S. J. 2005, A\&A, 432, 273

Kurucz, R. L. 1979, ApJS, 40, 1

Kwok, S., Purton, C. R., \& Fitzgerald, P. M. 1978, ApJL, 219, 125

Lagage, P., Jouan, R., Masse, P., et al. 1993, Proc. SPIE, 1946, 655

Lagadec, E., Mékarnia, D., de Freitas Pacheco, J. A., \& Dougados, C. 2005, A\&A, 433, 553

Leinert, C., Graser, U., Przygodda, F., et al. 2003, Ap\&SS, 286, 73

Leinert, C., van Boekel, R., Waters, L. B. F. M., et al. 2004, A\&A, 423, 537

Leuenhagen, U., Hamann, W.-R., \& Jeffery, C. S. 1996, A\&A, 312, 167

Matsuura, M., Zijlstra, A. A., Molster, F. J., et al. 2004, ApJ, 604, 791 Matsuura, M., Zijlstra, A. A., Molster, F. J., et al. 2005, MNRAS, 291

Meakin, C. A., Bieging, J. H., Latter, W. B., et al. 2003, ApJ, 585, 482

Miranda, L. F., Gómez, Y., Anglada, G., et al. 2001, Nature, 414, 284

Molster, F. J., Yamamura, I., Waters, L. B. F. M., et al. 1999, Nature, 401, 563

Pottasch, S. R. 1984, Astrophysics and Space Science Library, 107

Phillips, J. P. 2005, MNRAS, 361, 283

Przygodda, F., Chesneau, O., Graser, U., et al. 2003, Ap\&SS, 286, 85

Sahai, R., \& Nyman, L. 2000, ApJ, 538, L145

Sahai, R., \& Trauger, J. T. 1998, AJ, 116, 1357

Sahai, R., Zijlstra, A., Bujarrabal, V., \& Te Lintel Hekkert, P. 1999, AJ, 117, 1408

Sahai, R., Nyman, L.-A., \& Wootten, A. 2000, ApJ, 543, 880

Sorensen, P., \& Pollacco, D. 2004, Asymmetrical Planetary Nebulae III: Winds, Structure and the Thunderbird, ASP Conf. Ser., 313, 515

Waters, L. B. F. M., Cami, J., de Jong, T., et al. 1998, Nature, 391, 868 Waters, L. B. F. M., Beintema, D. A., Zijlstra, A. A., et al. 1998, A\&A, 331, L61

Waters, L. B. F. M., \& Molster, F. J. 1999, IAUS, 191, 209

Webster, L. B., \& Glass, I. S. 1974, MNRAS, 166, 491

Zijlstra, A. A., Gaylard, M. J., Te Lintel Hekkert, P., et al. 1991, A\&A, 243, L9 